\title{
Alpha-1-antitrypsin in Plasma Exosomes and Pericardial Fluid Exosomes Are Associated With Severity of Rheumatic Heart Disease
}

\section{Shruti Sharma}

PGIMER: Post Graduate Institute of Medical Education and Research

\section{Subendu Sarkar}

ESIC Medical College and Hospital Faridabad

Anuradha Chakraborti ( $\nabla$ chakravarti.anuradha@pgimer.edu.in )

PGIMER: Post Graduate Institute of Medical Education and Research https://orcid.org/0000-00018306-7558

\section{Research Article}

Keywords: Rheumatic heart disease, exosomes, serpin A1, mitral valve tissues, alpha-1 antitrypsin

Posted Date: January 31st, 2022

DOI: https://doi.org/10.21203/rs.3.rs-1274664/v1

License: (a) (1) This work is licensed under a Creative Commons Attribution 4.0 International License. Read Full License 


\section{Abstract}

Rheumatic heart is an autoimmune sequel of pharyngitis and rheumatic fever that leads to permanent heart valves damage, especially the mitral valves. The mitral valves, which are responsible for the binding of auto-antibodies during immune response generation, leads to valves scarring and eventually mitral valve dysfunction. Recently, exosomes (EXOs), the nano-sized-vesicles, which ranges in size from 30-150 $\mathrm{nm}$, are involved in various cardiovascular physiological and pathological processes. These vesicles are found in several body fluids such as plasma, serum and also in cell culture media. Exosomal cargo contains proteins, which are taken up by the recipient cells and modulates the cellular characteristics. The role of exosomal proteins in RHD is still obscure. Hence, the present study is designed to unveil the role of exosomal proteins in increasing disease severity during RHD. In this study, the exosomes were isolated from biological fluids (plasma and pericardial fluid) of RHD patients as well as from their respective controls. Protein profiling of these isolated exosomes revealed that alpha-1 antitrypsin is up-regulated in the biological fluids of RHD patients. The augmented levels of exosomal alpha- 1 antitrypsin, was further, validated in biological samples and mitral valve tissues of RHD patients, which was further correlated with the disease severity. These findings suggest that increased levels of exosomal alpha- 1 antitrypsin is responsible for increased severity during RHD.

\section{Introduction:}

Progression of rheumatic heart disease (RHD) in young adults is accomplished through the pathophysiology of group A streptococcus (GAS) infection and autoimmune rheumatic fever (Guilherme et al.,2004). RHD is a cardiovascular disease, where permanent heart valve damage takes place. Patient recovery at the end stage is possible only through the surgery or replacement of heart valve. However, poor diagnosis or lack of proper treatment or vaccines causes disease severity and even death.

Approximately, 30 to 40 million global cases of RHD have been recorded, with 300,000 deaths annually (Watkins et al.,2017). High mortality rates have been noticed in Oceania followed by south Asia and subSaharan Africa.

The exact mechanism behind development of RHD is poorly understood. However, most accepted hypothesis is based on the molecular mimicry due to cross reactivity of GAS and host proteins. Molecular mimicry results in the generation of immense autoimmune response during rheumatic fever, which facilitates the initiation of cardiac valve injury during RHD (Cunningham.,2014). Genetic susceptibility and profound cytokine secretion are associated with RHD pathophysiology (Guilherme et al.,2004). Besides, alteration of several host and pathogenic factors have also been reported to be linked with disease progression (Sarkar et al.,2016).

The autoimmune reaction generated during rheumatic fever has been reported to be accomplished by binding of streptococcal M protein N-terminus domain with CB3 region of host collagen IV (Dinkla et al.,2009). Recombinant M protein-mediated valvular tissue damage has been experimentally revealed in Lewis rat model (Quinn et al.,2001). However, several other autoantigens and host proteins have been 
found to be involved in the progression of RHD. Study conducted by Martins et al., demonstrated that increased tissue protein concentration of vimentin, lumican, and apolipoprotein A1 are associated with generation of autoimmunity in heart valve (Martins et al.,2017). Besides, auto-antigen cardiac myosin and adhesion molecules such as ICAM, VCAM has been found to induce the disease progression (Guilherme and Kalil.,2013).

High throughput advanced proteomic approaches allow to identify novel proteins and autoantigens involved in RHD pathophysiology. Plasma proteomic analysis performed by Mukherjee et al. have revealed a total of 34 differentially regulated proteins in rheumatic mitral stenosis out of which $50 \%$ of them are associated with inflammation and immune response (Mukherjee et al.,2014). Two-dimensional electrophoresis followed by mass spectrometry performed by Gao et al., alpha-1-antichymotrypsin, as a plasma maker associated with rheumatic valvular disease has been observed (Gao et al.,2013). The study based on immunohistochemistry and immunoblotting analysis has revealed the diverse distribution of lumican and collagen VI in rheumatic mitral valve (Martins et al.,2017). The study conducted on Lewis rat rheumatic mitral valve has shown 176 up-regulated and 119 down-regulated proteins through proteomic analysis. This study, further reported, GAPDH, CD9, myosin, collagen, and RAC1 as potential biomarkers of acute phase of RHD (Li et al.,2015).

Recently, exosomes gained tremendous attraction in the pathophysiology of cardiovascular diseases. Exosomes diameter ranging from 30 to $150 \mathrm{~nm}$ are membrane-bound extracellular vesicles released by certain cell types into blood or even different biological fluids such as pericardial fluid etc. Exosomes act as cargo, which carry cellular substances such as proteins, lipid, nucleic acids etc. to contribute targetspecific patho-physiological mechanisms (Stahl and Raposo., 2018). Studies based on exosomal long non-coding RNA profiling and miRNA-induced valvular damage have recently been reported in RHD (Luo et al.,2019). However, there is lack of information, which can reveal the involvement of exosomal proteins in the patho-physiology of RHD. Thus, the present study highlights the cumulative data obtained from proteomic analysis of blood as well as pericardial fluid exosomes. The findings are further intended to correlate with pathophysiology of RHD.

\section{Methods:}

\section{Sample collections:}

The present study is ethically approved by Institutional Ethics Committee (INT/IEC/2017/1453). Patient confidentiality is maintained throughout the study according to IEC guidelines. Informed consent has been taken from each participant. Clinically confirmed RHD patients $(\mathrm{N}=40)$ were included in the study. Blood, pericardial fluid (PF) were collected from the patients undergoing double valve replacement (DVR) and mitral valve replacement (MVR) surgery in the department of Cardiothoracic and Vascular Surgery (CTVS), Postgraduate Institute of Medical Education and Research (PGIMER), Chandigarh, India.

Pericardial fluid from Coronary artery bypass graft (CABGs) patients were collected as controls from the same department. Blood samples, as controls, were obtained from healthy individuals and from cadavers 
with no pathological or morphological heart disease, respectively. The patients suffering from any autoimmune disease, or any infectious disease and, not willing to give consent were excluded from the study. Plasma was separated centrifugation at $1500 \mathrm{rpm}$ for 20 minutes and $1 \mathrm{x}$ protease inhibitor (Sigma-Aldrich, USA) was added and the blood samples were stored at $-80^{\circ} \mathrm{C}$ until further use. After removal of debris from PF samples by centrifuged at $4000 \mathrm{~g}$ for 15 minutes, the samples were stored at $-80^{\circ} \mathrm{C}$ until further use.

\section{Exosomes isolation}

Exosomes were isolated from biological fluids (plasma and PF) with miRCury exosomes isolation kit (Qiagen, Germany), according to the manufacturer's instructions. Briefly, biological fluids were mixed with exosomes precipitation reagent at a ratio of $4: 1$ followed by overnight incubation at $4^{\circ} \mathrm{C}$. Further, centrifugation was carried out at $500 \mathrm{~g}$ for 5 minutes for plasma and $10,000 \mathrm{~g}$ for 30 minutes for PF, exosomes pellets were re-suspended in appropriate phosphate-buffered saline (GE Healthcare Life Sciences, USA). The exosomes pellets were used immediately or stored at $-80^{\circ} \mathrm{C}$ till further use.

\section{Exosomes characterization}

Exosomes were characterized with transmission electron microscopy (TEM), Zeta sizer and Western blot analysis.

\section{Transmission Electron Microscopy (TEM)}

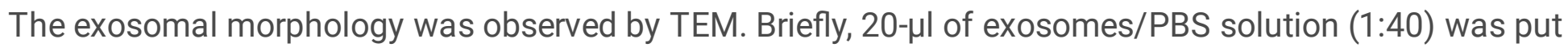
onto copper grids coated with carbon and allowed to stand for $1 \mathrm{~min}$. The staining of exosomes was done with $20 \mu \mathrm{l}$ uranyl acetate dihydrate (2\%) and left for $1 \mathrm{~min}$. Subsequently, the excess fluid was removed by using filter paper. The sample was air dried for 10 minutes and visualized under transmission electron microscope (H7650 Hitachi, Japan) (Jung and Mun.,2018).

\section{Zeta sizing}

Zeta sizer (Malvern paranalytical, UK, NIPER, Mohali) was used to measure the particle size diameter and percentage intensity of exosomes using the technique of Dynamic Light Scattering (DLS), according to the operating instruction. The sample was prepared by dispersing exosomes in PBS at 1:400 dilution.

\section{Western blot}

Exosomes were lysed in ice cold RIPA buffer. The measurement of protein concentration was done by BCA method (https://link.springer.com/protocol/10.1007/978-1-60327-259-9_3). The protein samples (50ug) were loaded on to SDS-PAGE and the protein bands from the gel were transferred $\left(80 \mathrm{~V}, 2.5 \mathrm{~h}, 4^{\circ} \mathrm{C}\right)$ onto Polyvinylidene fluoride membrane (PVDF) (GE Health care, USA) in the transfer buffer [25mM Tris/192mM Glycine ( $\mathrm{pH}$ 8.0)/20\% methanol] using mini-tank transfer unit (Bio-Rad mini Transblot ${ }^{\circledR}$ Cell, Bio-Rad, USA). After transfer, the blocking of membrane was done with $5 \%$ skimmed milk in Tris-buffered 
saline- $0.1 \%$ tween 20 (TBST overnight at $4^{\circ} \mathrm{C}$ ). Membrane was washed thrice for 5 min each with TBST to remove unbound blocking reagent. Primary antibody against CD63, (Biovision, USA, 1:100 and $\beta$-actin, (Biovision, USA, 1:200) was added and incubated at $37^{\circ} \mathrm{C}$ with shaking. Washing steps were repeated. Further, the membrane was incubated with secondary antibody (Biovision, USA, 1:2000), solution (HRP conjugated, Bangalore Genei, India) at $37^{\circ} \mathrm{C}$ with shaking. Again, washing steps were repeated. Membrane was exposed for 1 minute or longer for visualization and images were acquired in Protein Simple FlourChem M (Bio-Techne, USA).

\section{Protein profiling by liquid chromatography-mass spectrometry (LC-MS/MS)}

Total protein was extracted from RHD patients/CABG patients- PF exosomes and RHD patients/healthy controls- plasma exosomes. The LC-MS/MS analysis for the identification of differentially expressed exosomal proteins was commercially performed by Biologia Research India Pvt. Ltd. Briefly, Protein samples were trypsin digested and peptides extracted according to standard techniques (Bringans et al., 2008). Peptides were analysed by electrospray ionisation mass spectrometry using the Shimadzu Prominence nano HPLC system [Shimadzu] coupled to a 5600 TripleTOF mass spectrometer [Sciex]. Tryptic peptides were loaded onto an Agilent Zorbax 300SB-C18, 3.5 $\mu \mathrm{m}$ [Agilent Technologies] and separated with a linear gradient of water/acetonitrile/ $0.1 \%$ formic acid $(\mathrm{v} / \mathrm{v})$. Spectra were analysed to identify proteins of interest using Mascot sequence matching software [Matrix Science] with MSPnr100 database. Taxonomy: Homo sapiens (human) (April 2016; 338,672 sequences).

\section{Enzyme Linked Immuno Sorbent Assay (ELISA)}

The ELISA kit specific for alpha-1- antitrypsin protein was purchased from Biovision, USA, the Standard working solution was added(100 uL for each well), followed by addition of exosomal proteins from plasma, PF and mitral valves samples to the other wells (100 uL for each well). The plate was incubated for $90 \mathrm{~min}$ at $37^{\circ} \mathrm{C}$. The liquid was removed out of each well. Further, $100 \mu \mathrm{L}$ of Biotinylated Detection $\mathrm{Ab}$ working solution was added to each well and mixed up and the plate was incubated for 1 hour at $37^{\circ} \mathrm{C}$. The solution from each well was aspirated and $350 \mathrm{uL}$ of wash buffer was added to each well, soaked for 1-2 min and aspirated the solution from each well. Washing step were repeated 3 times. Further, $100 \mu \mathrm{L}$ of HRP Conjugate working solution was added to each well and incubated for $30 \mathrm{~min}$ at $37^{\circ} \mathrm{C}$. The solution from each well was aspirated and the washing process was repeated for five times. Next, $90 \mu \mathrm{L}$ of Substrate Reagent was added to each well and incubate for about $15 \mathrm{~min}$ at $37^{\circ} \mathrm{C}$ (protected the plate from light). Further, $50 \mu \mathrm{L}$ of stop solution was added to each well and the optical density of each well was determined with a micro-plate reader at $450 \mathrm{~nm}$. The protein levels were further correlated with the disease severity.

\section{Computational prediction of protein-protein interactions of Human Alpha-1 antitrypsin with heart autoantigens}

The FASTA sequences of human alpha1 antitrypsin and human heart autoantigens proteins (ECF13, Vimentin, Prolargin and Actin) were obtained from UniProt (https://www.uniprot.org/). In order to obtain a 
preliminary idea regarding the likelihood of interaction between human alpha1 antitrypsin (encoded by SERPINA1) and human heart autoantigens, the PSOPIA web server (https://mizuguchilab.org/PSOPIA/) was used. PSOPIA predicts protein-protein interactions using three sequence-based features; (I) sequence similarities to a known interacting protein pair $\left(\mathrm{S}_{\text {seq }}\right)$, (II) statistical propensities of domain pairs observed in interacting proteins $\left(S_{\text {dom }}\right)$ and (III) a sum of edge weights along the shortest path between homologous proteins in a PPI network $\left(S_{n e t}\right)$. So, for a protein-protein docking study was proceeded with the reported experimental structures of human alpha1 antitrypsin (PDB ID: 3NE4) and human vimentin (PDB ID: 1GK4) using the Cluspro Server. Before proceeding for protein-protein docking, InterProSurf server (http://curie.utmb.edu/prosurf.html) was used to predict the key residue patches on both the interaction partners, which might act as the interface residues

\section{Statistical analysis}

Statistical analysis was performed using SPSS software (version 22.0). All the experiments were carried out in triplicate and data were expressed as the mean \pm SD. To compare the data between two groups, Student's t-test was used and one-way analysis of variance (ANOVA) was used for comparison of more than two group means $p$-value $<0.05$ was considered statistically significant for all the experiments.

\section{Results:}

\section{Characterization of exosomes isolated from RHD patients:}

Exosomes were isolated from plasma of normal individuals and RHD patients. PF of CABG (control) and RHD patients were also taken for exosomes isolation. In all cases, exosomes have shown typical membrane-bound spherical morphology, as observed, through TEM (Fig. 1). The size distribution of exosomes based on diameter and intensity were measured by Zeta sizer. Plasma exosome from normal individuals showed typical particle sizes ranging from 10 to $100 \mathrm{~nm}$ having peak intensity at size of $50 \mathrm{~nm}$ (Fig. 1A). Interestingly, plasma exosomes from RHD patients showed two peak intensities at size of $20 \mathrm{~nm}$ and 50nm (Fig. 1B). Similar to normal individuals, PF exosomes from control exhibited typical particle size distribution ranging from 10 to $100 \mathrm{~nm}$ (Fig. 1C). On the other hand, PF exosomes from RHD patients showed particle size ranging from 100-150nm (Fig. 1D). Further to confirm, exosomes were characterized through Western blot using exosome-specific surface marker CD63. Plasma/PF of control and RHD showed $65 \mathrm{KDa}$ exosomal protein bands on Western blot (Fig. 1E).

\section{Identification of exosomal proteins by LC-MS/MS:}

PF exosomal protein profiles through LC-MS/MS were performed in RHD, where CABG PF exosomes were used as control. Identified proteins in RHD with fold change>1 were recorded for apolipoprotein A-I, immunoglobulin kappa constant, complement $\mathrm{C} 1 \mathrm{r}$ subcomponent, keratin, type II cytoskeletal 1, alpha-1antitrypsin, immunoglobulin heavy constant gamma 2, Immunoglobulin heavy constant gamma 4, immunoglobulin heavy constant gamma 1, complement C1s subcomponent, and immunoglobulin heavy constant alpha 1 (Fig. 2A.). Similar to PF exosome, LC-MS/MS were also done for RHD plasma 
exosomes, where healthy plasma was used as control. Identified proteins in RHD plasma exosomes with fold change $>1$ were immunoglobulin heavy constant gamma 3 , immunoglobulin heavy constant mu, immunoglobulin heavy constant alpha 1, inter-alpha-trypsin inhibitor heavy chain $\mathrm{H} 4$, alpha-1-antitrypsin, immunoglobulin lambda variable 3-9, complement factor I, complement component $\mathrm{C}$, immunoglobulin heavy variable 3-49, and histidine-rich glycoprotein (Fig. 2B). The exosomal alpha-1 antitrypsin was selected as a common protein with elevated levels via LC/MS/MS protein profiling in plasma exosomes and PF exosomes. Various de-regulated exosomal proteins were found to be the common proteins in plasma as well as PF exosomes, such as, Apo-lipoproteins, Fibrinogen-alpha chain, Immunoglobulin Heavy Chains, however, none of these proteins showed the protein fold change $>1$. Apo-lipoproteins (AP01) levels was considerably augmented in PF exosomes with fold change $>2$, whereas the levels of AP01 declined in plasma exosomes. Therefore, apo-lipoproteins were not taken into consideration for further study. Notably, alpha-1 antitrypsin levels were estimated for further validation in the serum samples, PF samples, plasma exosomes, PF exosomes and mitral valve tissues from RHD patients and their respective controls. The significant up-regulated expression levels of exosomal protein alpha-1 antitrypsin has been found in all RHD patients' samples. Further, these findings were correlated with the disease severity (mitral valve regurgitation) during RHD and it was found that severity grades significantly increased in all RHD patients' samples as compared to their respective controls.

\section{Concentration of alpha-1-antitrypsin in pathological samples and control individuals:}

Alpha-1-antitrypsin was measured in different pathological samples including PF, plasma, mitral valve tissues, PF-exosomes, and plasma exosomes. In all cases Alpha-1-antitrypsin concentration was estimated to be high in RHD patients compared to controls.(Fig. 3A.). Notably, alpha-1-antitrypsin concentration was maximally found in mitral valve tissues of RHD patients $(69.10 \pm 13.65 \mathrm{mg} / \mathrm{ml})$ compared to control tissues $(42.44 \pm 2.39 \mathrm{mg} / \mathrm{ml})(p=0.003)$. Whereas, alpha-1-antitrypsin concentration of plasma exosomes showed, $39.58 \pm 21.07 \mathrm{mg} / \mathrm{ml}$ in RHD and $14.25 \pm 8.37 \mathrm{mg} / \mathrm{ml}$ in healthy individuals $(p=0.04)($ Table- 1$)$.

Table 1

Concentration of alpha-1-antitrypsin in different pathological samples.

\begin{tabular}{|llllll|}
\hline Sr. No. & Sample resources & \multicolumn{2}{l}{ Study groups } & \multicolumn{2}{l|}{$p_{\text {-value }}$} \\
\cline { 3 - 6 } & & RHD & CABG & Healthy & \\
\hline 1 & PF-exosome & $26.25 \pm 25.23$ & $13.90 \pm 6.82$ & - & 0.32 \\
\hline 2 & Serum-exosome & $39.58 \pm 21.07$ & - & $14.25 \pm 8.37$ & 0.04 \\
\hline 3 & PF & $25.68 \pm 28.24$ & $9.2 \pm 8$ & - & 0.09 \\
\hline 4 & Serum & $33.39 \pm 37.31$ & - & $9.77 \pm 6.01$ & 0.06 \\
\hline 5 & Mitral valve & $69.10 \pm 13.65$ & - & $42.44 \pm 2.39$ & 0.003 \\
\hline
\end{tabular}

( ${ }^{*} p<0.05$ was considered as significant) 
Concentration of alpha-1-antitrypsin based on pathological grades of mitral valves regurgitation:

Alpha-1-antitrypsin concentration in RHD patients and control was also evaluated based on pathological grade of mitral valves regurgitation. The pathological grades of mitral valve regurgitation were classified as "severe", "moderate" and "absent". In all study groups, alpha-1-antitrypsin concentration were found to be high in severe regurgitation followed by other pathological grades (Fig. 3B). Mean alpha-1-antitrypsin concentration was noticed maximum in RHD patients valvular tissues $(76.02 \mathrm{mg} / \mathrm{ml})$ having "severe" mitral regurgitation compared to "moderate" $(53 \mathrm{mg} / \mathrm{ml})$ and "absent" $(34 \mathrm{mg} / \mathrm{ml})$ severity grades.

\section{Protein-protein interactions of human alpha-1 antitrypsin with heart auto antigens:}

The predictions obtained from PSOPIA showed that except for human ECF13, all the proteins have at least one score $>0.5$, indicating some probability to interact with human alpha1 antitrypsin. The human vimentin protein showed high Sdom, Snet and overall Sall scores for interacting with the alpha1 antitrypsin. The probabilities of protein-protein interaction of human alpha1 antitrypsin with the heart autoantigens is shown in Fig. 4A (i-v). The crystal structure for human vimentin is also available in Protein Data Bank. InterProSurf predicted N230-C232 and W238 of serpin (3NE4) and N350-N357 of vimentin (1GK4) as residues with high probabilities for protein function and protein-protein interaction (Fig. 4B, 4C).

Residue patches of human Alpha1 antitrypsin and Vimentin with high probabilities for participation in protein-protein interactions, as predicted by InterProSurf. Now based on these residues, docking calculation was submitted in ClusPro (https://cluspro.bu.edu/home.php).

ClusPro rotates the ligand protein with 70,000 rotations along with translation in $x, y, z$ directions with respect to the receptor on a grid and the translation with the best score from each rotation was chosen. The best scoring 1000 ligand poses were retained and were clustered with a 9 angstrom C-alpha rmsd radius. For analysis, the cluster centre of the largest cluster with 342 poses was taken, Table 2 shows top 10 clusters from ClusPro analysis and the number of structures in each of these clusters.

Table 2

Clusters from ClusPro and the number of structures in each these clusters

\begin{tabular}{|lllllllllll|}
\hline Cluster & $\mathbf{0}$ & $\mathbf{1}$ & $\mathbf{2}$ & $\mathbf{3}$ & $\mathbf{4}$ & $\mathbf{5}$ & $\mathbf{6}$ & $\mathbf{7}$ & $\mathbf{8}$ & $\mathbf{9}$ \\
\hline Members & 340 & 221 & 122 & 113 & 105 & 62 & 62 & 61 & 53 & 49 \\
\hline
\end{tabular}

Analysis of the structure representative of Cluster 0 showed several non-covalent interactions between the key residue patches between human alpha1 antitrypsin and vimentin (Table 2). Lys233 of human alpha1 antitrypsin makes two $\mathrm{H}$-bonds and a salt bridge interaction with the oppositely charged Glu354 of vimentin. His231 and Trp238 makes $\pi-\pi$ stacking interactions with Trp551 of vimentin. These interactions showed that the complex is stabilized both by polar and hydrophobic types of interactions. 
Non-covalent interactions between the key residue patches between human alpha1 antitrypsin and vimentin are shown in Fig. 4(D).

\section{Discussion:}

Molecular mimicry of several host and group A streptococcus (GAS) proteins play important roles to deteriorate mitral valves, leading to progression of RHD (Guilherme et al.,2004). Hence, identification of causative proteins and binding efficacy of these proteins are pivotal to understand mechanisms behind mitral valve damage in RHD. Identification of several proteins by high throughput proteomic techniques have already been reported in RHD (Mukherjee et al.,2014). However, role of circulatory exosomes and exosomal proteins in RHD pathophysiology are yet to be unfold. Exosomal research has gained attention in various diseases including cardiovascular diseases. Hence, the involvement of exosomes in mitral valve dysfunction during RHD development has been unfurled in the study. Further, we extended the focus towards exosomal proteins in the study to evaluate the role of proteins in mitral valve dysfunction during RHD. Total protein extracted from plasma exosomes and PF exosomes were analysed for LCMS/MS.

In this study, isolated exosomes were morphologically characterized by TEM, where membrane-bound spherical shaped structures were visualized (Jung et al.,2018). Additionally, $\mathrm{CD} 63^{+}$exosomes were also confirmed by Western blot (Fig. 1). LC-MS/MS protein profiling in RHD plasma and PF, exosomes revealed a-1-antitrypsin as a common protein with up-regulated expression levels. Alpha-1 antitrypsin is a serpin superfamily acute phase protein and responsible for inhibiting multiple proteases (Wettstein et al.,2021). However, it acts majorly as serine proteinase inhibitor and abundantly present in plasma. Thus, it is often named as alphal-proteinase inhibitor or alpha1-antiproteinase. The SERPINA1 gene is responsible for encoding alpha- 1 antitrypsin in humans.

The quantitation of alpha- 1 antitrypsin revealed its elevation with increasing severity during RHD. It was fascinating to understand whether the exosomal alpha- 1 antitrypsin in circulation possesses binding with the heart auto-antigens. We have already reported various heart auto-antigens such as EFC13, vimentin, prolargin and actin in RHD patients. The computational prediction of protein-protein interactions of alpha1 antitrypsin with heart auto-antigens was bioinformatically analysed. Except for human ECF13, all of other proteins were predicted by PSOPIA had at least one score $>0.5$. It indicates a possibility of interaction with human alpha-1 antitrypsin. For interacting with the alpha-1 antitrypsin, the human vimentin protein showed good Sdom, Snet, and overall Sall scores. Clus pro results for docking revealed that there are several non-covalent interactions between the key residue patches between human alpha1 antitrypsin and vimentin. Hydrogen bonds, $\pi-\pi$ stacking, salt bridges were the types of interaction between the two proteins. It has been previously reported that vimentin levels are up-regulated during RHD. Previous studies also identified the deregulated proteins and auto-antigens such as PDIA3, HSPA5, biglycans and prolargin in mitral valve tissues of RHD patients (Fae KC et al., 2008; Martins Cde 0., 2014). 
We have found that vimentin, confirming prior findings that these proteins are autoantigenic ((Fae KC et al., 2008). Vimentin antibodies have been found in a variety of inflammatory and autoimmune illnesses, including rheumatoid arthritis and allograft rejection (Davidson A., 2014). A study also found that vimentin, a cytoskeletal protein has a role in wound healing, which is normally found in the cytoplasm of mesenchymal cells, is expressed on the surface of damaged cells. Furthermore, when apoptosis is induced, this cytosolic antigen gets confused, exposing cryptic epitopes to immune cells (Rose ML., 2013).

Profound inflammation and inflammatory response are generated during autoimmunity. Alpha-1 antitrypsin acts as an anti-inflammatory protein, which is reported to be beneficial in several autoimmune diseases such as rheumatoid arthritis and systemic lupus erythematosus (Song et al.,2018). However, its level is increased in response of inflammation and tissue damage. Having anti-neutrophil protease activity alpha- 1 antitrypsin is involved in inhibiting neutrophil serine protease and serine elastase. Cumulative data from different studies suggest and hypothesize that alpha- 1 antitrypsin can be selfassembled by cleaving, oxidizing itself, which regulates neutrophil functions as well as neutrophilmediated inflammation. According to our data, the presence of alpha- 1 antitrypsin in plasma and pericardial fluid as exosomal protein depicts easy accessibility of this protein toward heart valves. It has been reported that alpha-1 antitrypsin polymers have proinflammatory action, which allows neutrophil migration and causes connective tissue damage in lung (Parmar et al.,2002). The present study showed the binding efficiency of alpha- 1 antitrypsin with mitral valve-derived protein vimentin. Alpha- 1 antitrypsin itself or its interaction with vimentin may facilitate neutrophil chemotaxis and neutrophil recruitment towards inflamed mitral valve during RHD (https://www.atsjournals.org/doi/10.1513/AnnalsATS.201509634KV;https://respiratory-research.biomedcentral.com/articles/10.1186/rr78). Alpha-1 antitrypsin in mitral valve of RHD patients was detected to be high. Study with histopathological evidences revealed that neutrophilic migration takes place into mitral valve during permanent heart valve damage in RHD (Guilherme et al.,2004). Alpha-1 antitrypsin is maximally found in neutrophil as granular neutrophilic protein. It may be explained that accumulation of migrated neutrophils are the major source of higher level of alpha-1 antitrypsin in rheumatic mitral valve tissues.

\section{Conclusion:}

In conclusion, elevation of alpha-1 antitrypsin in plasma, pericardial fluid, and mitral valve tissue of RHD patients is associated with disease severity. High concentration of alpha- 1 antitrypsin in plasma and pericardial fluid exosomes, further, demonstrates the plausible role of exosomes in pathophysiology of RHD. Alpha-1 antitrypsin and vimentin interaction may have the potential role in infiltrating neutrophil into inflamed mitral valve tissues, which depicts the possibilities of valve tissue deterioration and dysfunction in RHD. The study might open gateways for the future research in order to better understand the RHD pathogenesis.

\section{Declarations:}


Availability of Data and Material:

Yes

\section{Author's Contribution:}

AC conceived the idea, $\mathrm{SS}^{\mathrm{a}}$ designed the framework, performed the experimental work and drafted the manuscript. $S S^{b}$ analysed the data and contributed in drafting the manuscript. The author approved the final version of the manuscript.

Code Availability:

None

Funding:

The funding was received by Indian Council of Medical Research (ICMR), Delhi, India.

Compliance with Ethical Standards:

Yes

Conflicts of Interest/Competing Interests:

None.

Ethics Approval:

Approval for the study was granted by Institutional Ethics Committee, Postgraduate Institute of Medical Education and Research, Chandigarh, (INT/IEC/2017/1437; Date: 04.12.2017).

Consent to Participate:

Informed consent was obtained from all individual participants included in the study.

Consent for Publication:

Yes

\section{References:}

1. Guilherme L, Cury P, Demarchi LMF, Coelho VN, Abel LC, Lopez AP et al. Rheumatic Heart Disease Proinflammatory Cytokines Play a Role in the Progression and Maintenance of Valvular Lesions. American Journal of Pathology. 2004; 165:1583-1591. 
2. Watkins DA, Johnson CO, Colquhoun SM, Karthikeyan G, Beaton A, Bukhman G et al. Global, Regional, and National Burden of Rheumatic Heart Disease, 1990-2015, The New England Journal of Medicine. 2017;377:713-22.

3. Cunningham MW. Rheumatic Fever, Autoimmunity and Molecular Mimicry: The Streptococcal Connection. International Reviews of Immunology. 2014 ; 33: 314-329.

4. Sarkar S Chopra S, Rohit MK, Banerjee D Chakraborti A et al Vitamin D regulates the production of vascular endothelial growth factor: A triggering cause in the pathogenesis of rheumatic heart disease? Medical Hypotheses 2016;95:62-66.

5. Dinkla K, Talay SR, Mo“ rgelin M , Graham RMA, Rohde M, Nitsche-Schmitz DP et al. Crucial Role of the CB3-Region of Collagen IV in PARF Induced Acute Rheumatic Fever. PLoS One. 2009; 4(3): e4666.

6. Quinn A, Kosanke S and Fischette VA. Induction of Autoimmune Valvular Heart Disease by Recombinant Streptococcal M Protein.. American Society for Microbiology. Infection and immunity. 2001; 69: 4072-4078.

7. Martins CO, Demarchi L, Ferreira FM, Pomerantzeff PA, Brandao C, Sampaio RO et al. Rheumatic Heart Disease and Myxomatous Degeneration: Differences and Similarities of Valve Damage Resulting from Autoimmune Reactions and Matrix Disorganization. PLoS One. 2017;12:e0170191.

8. Guilherme L, and Kalil J. Rheumatic heart disease: molecules involved in valve tissue inflammation leading to the autoimmune process and anti-S. pyogenes vaccine. Frontiers in immunology.2013; 4: 352.

9. Mukherjee S, Jagadeeshaprasad MG , Banerjee T, Ghosh SK, Biswas M , Dutta S et al. Proteomic analysis of human plasma in chronic rheumatic mitral stenosis reveals proteins involved in the complement and coagulation cascade. Clinical Proteomics. 2014, 11:35.

10. Gao G, Xuan C, Yang Q,Liu XC,Liu ZG,He GW. Identification of Altered Plasma Proteins by Proteomic Study in Valvular Heart Diseases and the Potential Clinical Significance. Plos One, 2013;8:e72111.

11. Martins CO, Demarchi L„, Ferreira FM, Pomerantzeff PA, Brandao C, Sampaio RO et al. Rheumatic Heart Disease and Myxomatous Degeneration: Differences and Similarities of Valve Damage Resulting from Autoimmune Reactions and Matrix Disorganization. PLoS One. 2017;12:e0170191.

12. Li W, Zeng Z, Gui C , Zheng H, Huang W, Wei $\mathrm{H}$ et al. Danping Gong. Proteomic analysis of mitral valve in Lewis rat with acute rheumatic heart disease. International Journal of Clinical and Experimental Pathology 2015;8:14151-14160.

13. Stahl PD , Raposo G. Exosomes and extracellular vesicles: the path forward. Essays in Biochemistry. 2018;62:119-124.

14. Luo Y, Huang L , Luo W, Ye S and Hu Q. Genomic analysis of IncRNA and mRNA profiles in circulating exosomes of patients with rheumatic heart disease. Biology Open. 2019; 8: bio045633.

15. Jung MK and Mun JY. Sample Preparation and Imaging of Exosomes by Transmission Electron Microscopy. Journal of Visualised Experiments.2018;131:e56482. 
16. Walker JM. The Bicinchoninic Acid (BCA) Assay for Protein Quantitation. The Protein Protocols Handbook : 11-14.

17. Bringans S, Eriksen S, Kendrick T, Gopalakrishnakone P, Livk A, Lock R, et al. Proteomic analysis of the venom of Heterometrus longimanus (Asian black scorpion). Proteomics. 2008; 8: 1081-96.

18. Wettstein L Weil T, Münch J.Alpha-1 antitrypsin inhibits TMPRSS2 protease activity and SARS-CoV-2 infection. Nature Communications.2021; 12:1726.

19. Fae KC, Diefenbach da Silva D, Bilate AM, Tanaka AC, Pomerantzeff PM, Kiss MH, et al. PDIA3, HSPA5 and vimentin, proteins identified by 2-DE in the valvular tissue, are the target antigens of peripheral and heart infiltrating $T$ cells from chronic rheumatic heart disease patients. $J$ Autoimmun. 2008; 31: 136-41.

20. Martins Cde O, Santos KS, Ferreira FM, Teixeira PC, Pomerantzeff PM, Brandão CM, et al. Distinct mitral valve proteomic profiles in rheumatic heart disease and myxomatous degeneration. Clin Med Insights Cardiol. 2014; 8: 79-86.

21. Davidson A. Autoimmunity to vimentin and lupus nephritis. Arthritis Rheumatol. $2014 ; 66(12)$ : 3251-3254.

22. Rose ML. Role of anti-vimentin antibodies in allograft rejection. Hum Immunol. 2013; 74: 1459-62.

23. Song S. Alpha-1 Antitrypsin Therapy for Autoimmune Disorders. Chronic Obstr Pulm Dis. 2018;5(4):289-301.

24. Parmar JS, Mahadeva R, . Reed BJ, Farahi N, Cadwallader KA, Keogan MT et al. Polymers of $a_{1^{-}}$ Antitrypsin Are Chemotactic for Human Neutrophils A New Paradigm for the Pathogenesis of Emphysema. American Journal of Respiratory Cell and Molecular Biology2002.Biol;26:723-730.

25. Leukocyte adhesion and recruitment, and alpha-1-antitrypsin deficiency: a report from ATS 2001, May 18-23, San Francisco lan Woolhouse, Meeting report. Respiratory Research. 2001.

\section{Figures}


(A) Plasma exosomes from normal individuals
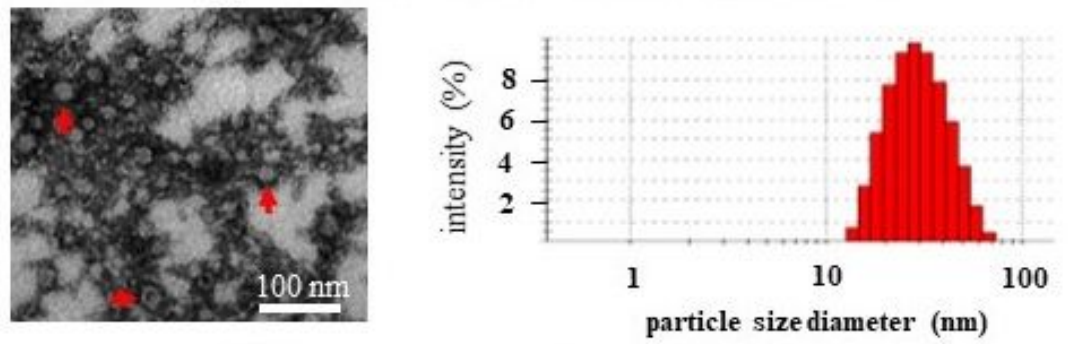

(B) Plasma exosomes from RHD patients
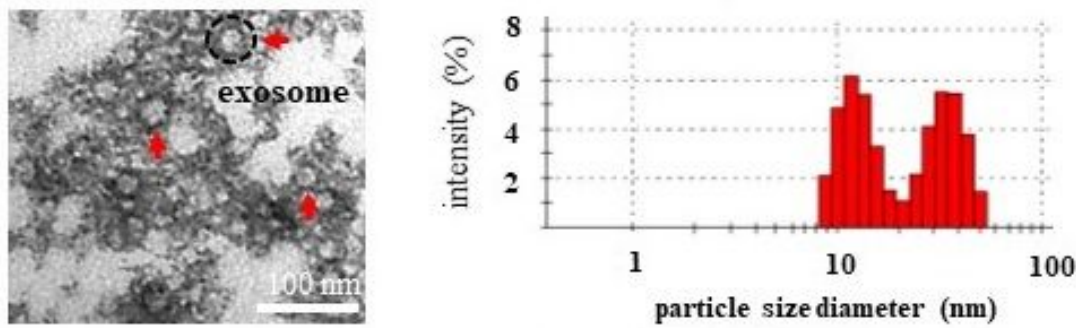

(C) PF exosomes from CABG patients
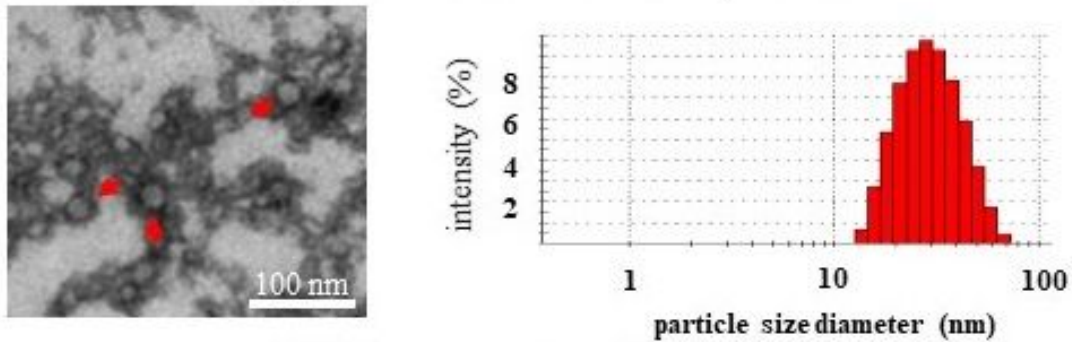

(D) PF exosomes from RHD patients
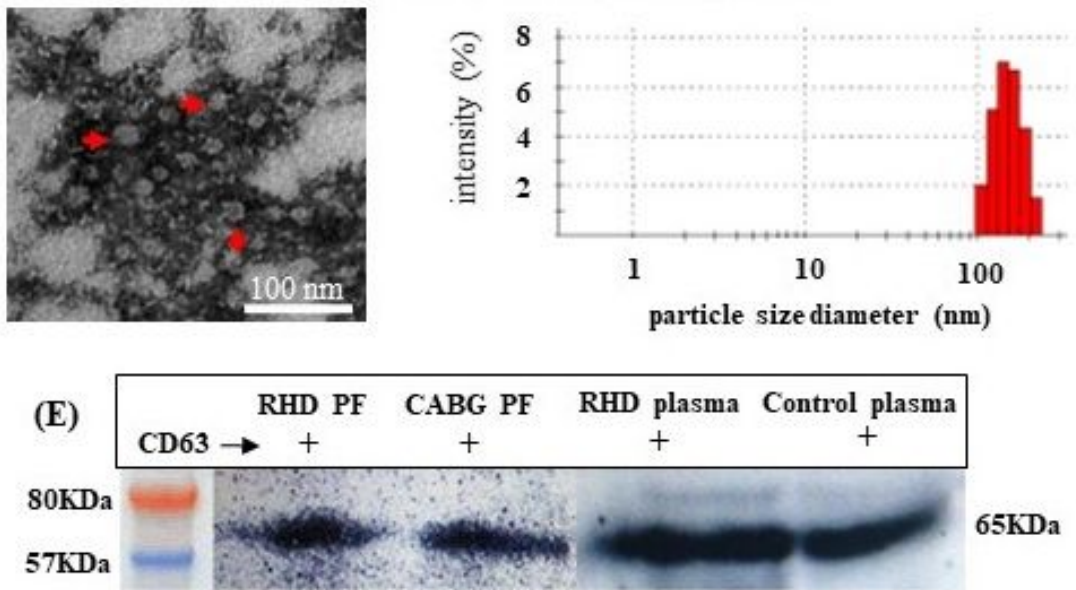

Fig. 1.

\section{Figure 1}

Characterization of plasma exosomes by TEM and zeta sizing from(A) normal individuals (B) RHD patients (II) PF exosomes from (C) CABG patients (controls) and (D) RHD patients (E) Western blot analysis of plasma and PF exosomes from RHD patients and their respective controls. 
(A)

RHD vs. CABG PF

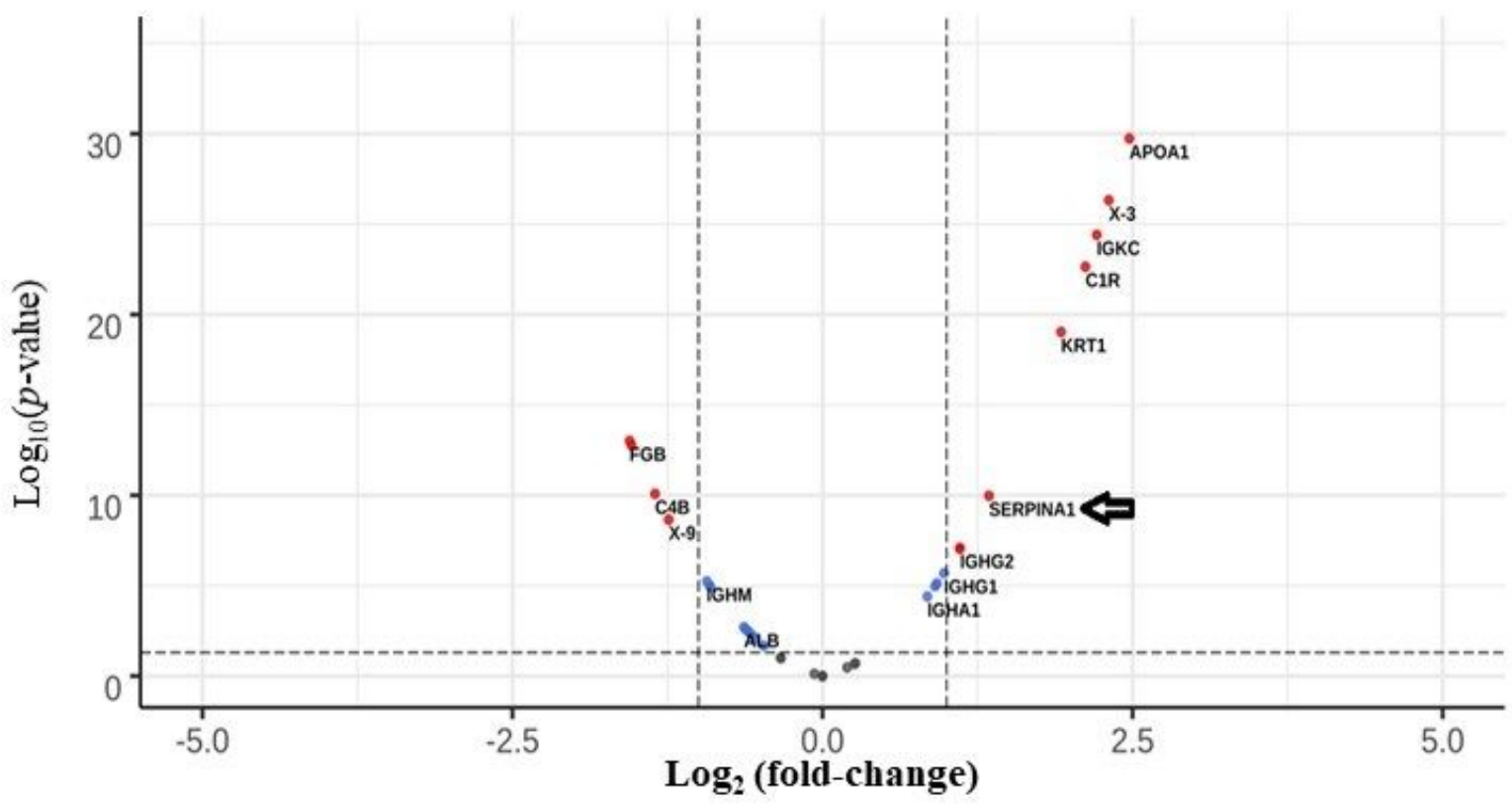

(B)

\section{RHD vs. healthy plasma}

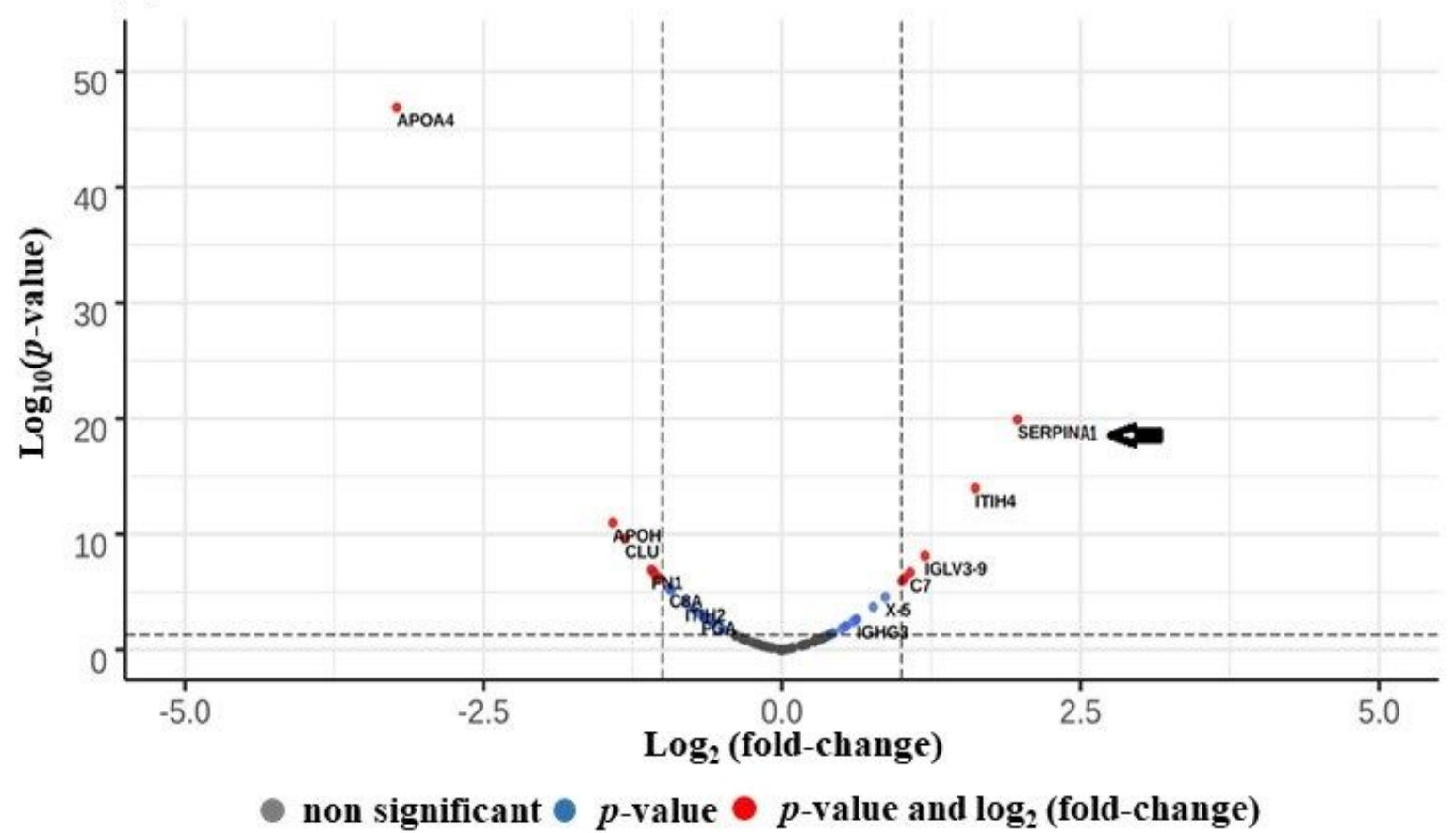

Fig. 2.

Figure 2

Volcano plot representing differential expression (log Fc) in: (A) exosomal PF (B) exosomal plasma proteins in RHD patients in comparison to their respective controls. 
(A)

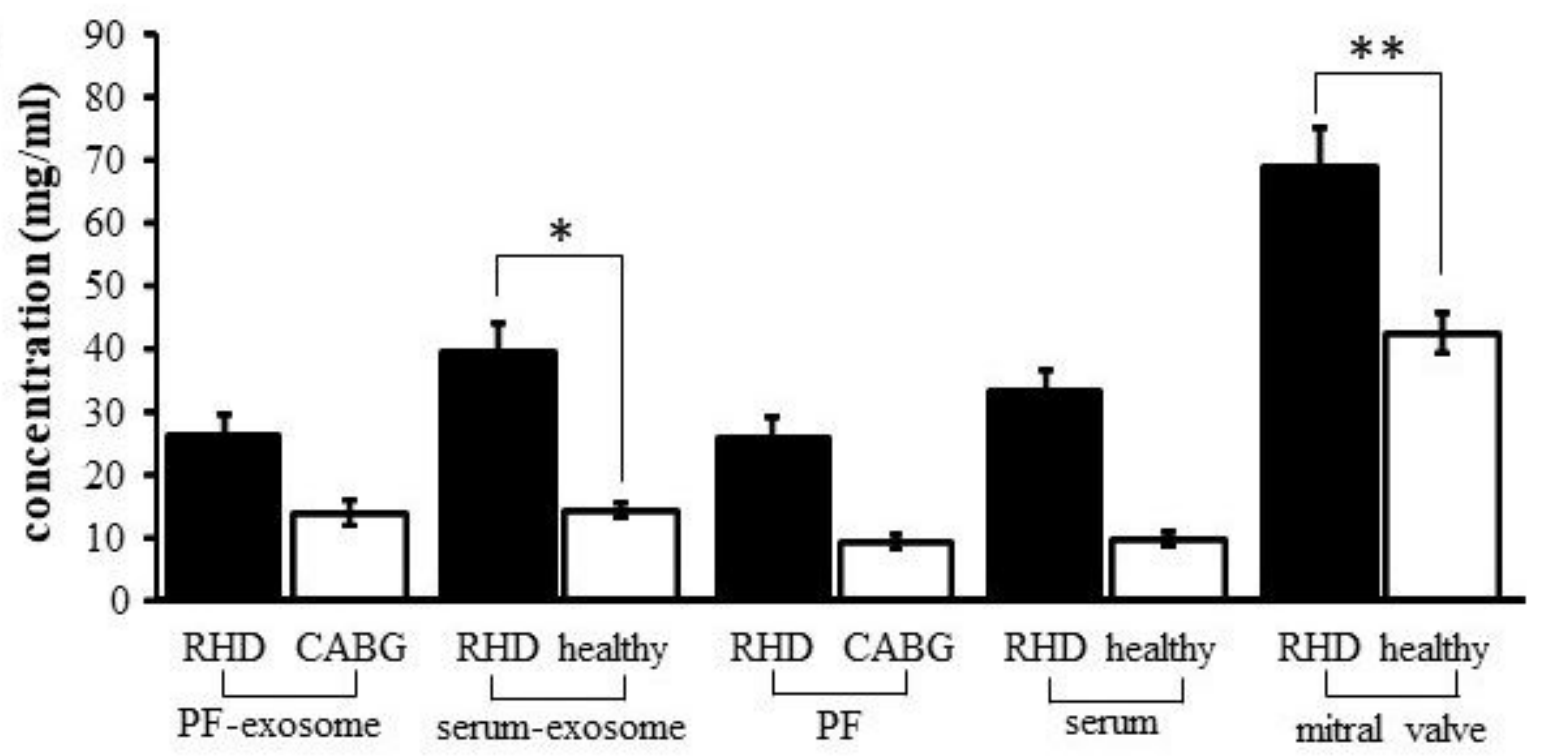

(B)

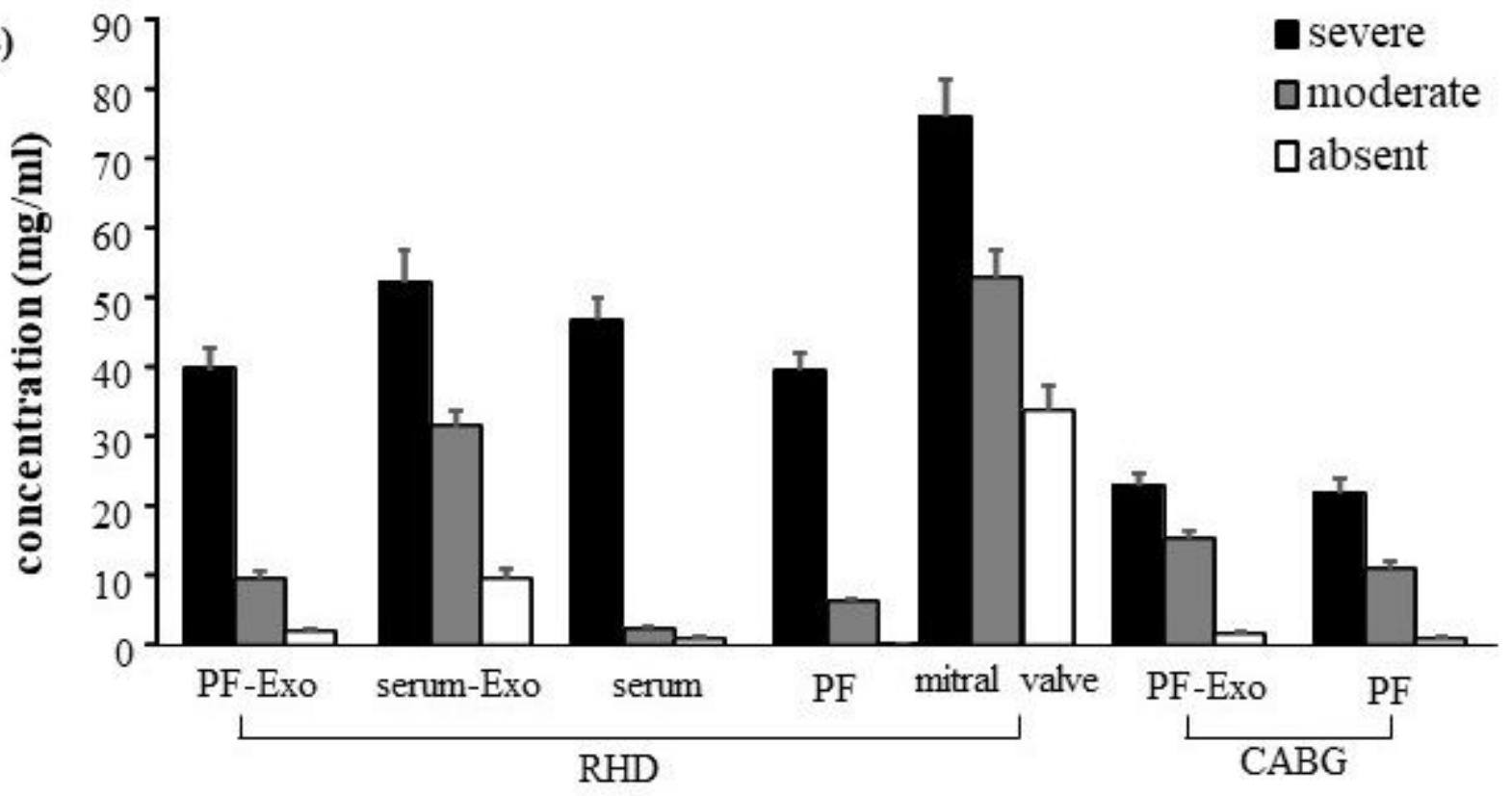

Fig. 3.

Figure 3

(A) Alpha-1-antitrypsin protein levels in PF exosomes, plasma exosomes, PF, plasma, mitral valve tissues from RHD patients and controls. All experiments were repeated three times. Each bar represents the mean \pm S.D. Statistical analysis was carried out by student's t-test. ${ }^{*} P<0.05,{ }^{\star} \mathrm{P}<0.01$, ${ }^{\star \star *} \mathrm{P}<0.001$ compared to control (B) Association of the levels of de-regulated protein (alpha-1-antitrypsin) with disease severity. All experiments were repeated three times. Each bar represents the mean \pm S.D. Statistical analysis was carried out by one way ANOVA, ${ }^{\star} \mathrm{P}<0.05,{ }^{*} \mathrm{P}<0.01,{ }^{\star \star *} \mathrm{P}<0.001$ compared to control. 
Fig. 4.

\section{Figure 4}

(A) Probability scores of bindings alpha-1-antitrypsin with heart-auto antigens $(B, C)$ Predicted high interacting residues of alpha-1-antitrypsin and vimentin (D) Non-covalent interactions between the key residue patches between human alpha-1-antitrypsin and Vimentin. 